\title{
Fauna, ecology and taxonomy of helminths of cypriniformes fish water bodies of Uzbekistan
}

\author{
$F$ Safarova $^{1, *}, F$ Akramova $^{2}, D$ Azimov $^{2}, B$ Qahramanov $^{1}$, and $D$ Gulomjonov ${ }^{1}$ \\ ${ }^{1}$ Tashkent State Agrarian University, University str. 2, Tashkent, 100140 Uzbekistan \\ ${ }^{2}$ Institute of Zoology, Uzbek Academy of Sciences, Bogishamol str. 232, Tashkent, 100053 \\ Uzbekistan
}

\begin{abstract}
Due to intensive human activities related to the use of water resources, has undergone substantial qualitative and quantitative changes of biocenosis and the fish community. This inevitably arise parasitic fish diseases that lead to a decrease in the number of valuable species and the deterioration of the epizootic situation reservoirs. Fauna of parasitic worms of Cypriniformes ponds diverse river of the Syrdarya shown 49 species. 18 species belongs to the class Trematoda, Cestoda class represented by 13 species, class Acanthocephala - 4, and the class Nematoda - 14. Analysis of biological properties and ecological of specialty parasitic worms Cypriniformes allows three types of communities: helminths ( 25 species), parasitizing Cypriniformes as definitive hosts; helminths (19 species), parasitizing Cypriniformes as intermediate hosts; - helminths (6 species), parasitizing Cypriniformes as a reservoir (=paratenetic) hosts.
\end{abstract}

\section{Introduction}

Helminths, certainly, one of the most popular objects of study of the fauna of the Syrdarya River Basin [1]. The Syrdarya River Basin is a natural geographic complex cross-border area, in which there are a variety of environmental conditions ponds. Currently, the Syrdarya basin, a large number of large reservoirs of complex use of hundreds of thousands of hectares of area $[1,2]$. Reservoirs are a new type of water bodies, characterized by specific and ecological conditions $[2,3]$.

Due to intensive human activities related to the use of water resources, has undergone substantial qualitative and quantitative changes of biocenosis and the fish community. This inevitably arise parasitic fish diseases that lead to a decrease in the number of valuable species and the deterioration of the epizootic situation reservoirs. Parasitic diseases of fish not only cause significant economic losses associated with a decrease in fish productivity, and they are dangerous to human health [2-4].

For information on the fauna of fish helminthes in the region are reflected in the works [1-6]. To date, data from previous researchers significantly out of date, as confirmed by the recent resumption of studies helminthes of fauna of the region [6].

Based on the above it is actually a detailed study to ichthyo-parasitological of the current state of helminthes communities of their distribution within the named pool.

* Corresponding author: feruzasafarova@mail.ru 


\section{Materials and methods}

Stationary studies were conducted in the period 2009-2020 years in the water bodies of the Syrdarya basin (the Syrdarya River, the Chirchik River, Aydar-Arnasay lake system, Tuyabuguz reservoir and fish farms, "Balikchi", "Damachi" and "Tashkent fish farm"), in Syrdarya, Tashkent and Djizakh region.

Collection and study of helminths of fish conducted by well-known methods [7-12]. Besides, we carried out incomplete dissections of 1338 fish individuals and prepared 1250 temporary and permanent whole mounts.

The cameral treatment and identification of trematode species was carried out at the Laboratory of General Parasitology of the Institute of Gene Pool of Plants and Animals of Uzbek Academy of Sciences. Helminth species were identified by using the Reference Guide of Freshwater Fishes and the Catalogues [7-14].

The studies were conducted using a microscope type «Olympus CK 2". The preparations were examined under the microscope MBI-3 and MBI-4, while the drawings were produced using the drawing tubes RA-4 and RA-5.

\section{Results and discussion}

We found that the helminths of Cypriniform fish the basin of the Syrdarya (within Uzbekistan) currently presents 49 species: 18 species of Trematoda, 13 of Cestoda. Representatives of the class Nematoda consist of 14 species and Acanthocephala - 4 species.

Class Trematoda represented in the studied basin us 18 species belonging to the families of 9 and 5 orders. The most numerous representatives of the order Strigeida La Rue, 1926 10 species. As part of other groups marked only 1 or 2 kinds of trematodes (Table 1).

Representatives of the four groups Caryophyllida, Pseudophyllida, Proteocephalida and Cyclophyllida class Cestoda, in the investigated region, there are 13 species of fish orders Cypriniformes. The most widespread are the families Caryophyllaeidae Leuckart, 1878 and Dilepididae Fuhrmann, 1907 (four species each).

The Acanthocephala included four species: Neoechinorhynchus rutili Müller, 1780, Pomphorhynchus laevis Müller, 1776, Acanthocephalus lucii Müller, 1776 and A. anguillae Müller, 1780. The recorded acanthocephalan species, except for A. lucii, had been previously found in the Cypriniform fish in the mouth of the Syrdarya River [1-3].

Considerable diversity of species in the waters of the middle reaches of the Syrdarya is characterized by the class Nematoda (14 species) belonging to four orders. Trichocephalida Skrjabin et Schulz, 1928 is represented by one species, Capillaria tomentosa Dujardin, 1843, which was found in most Cypriniformes in natural and artificial water bodies. The highest species diversity was observed in the Spirurida Chitwood, 1933, for which we recorded eight species.

Table 1. Taxonomic composition of helminths parasitizing Cypriniformes in the studied area.

\begin{tabular}{|c|c|c|c|}
\hline Class & Order & Family & Species \\
\hline Trematoda & Sanguinicolida & Sanguinicolidae & Sanguinicola inermis \\
\hline
\end{tabular}




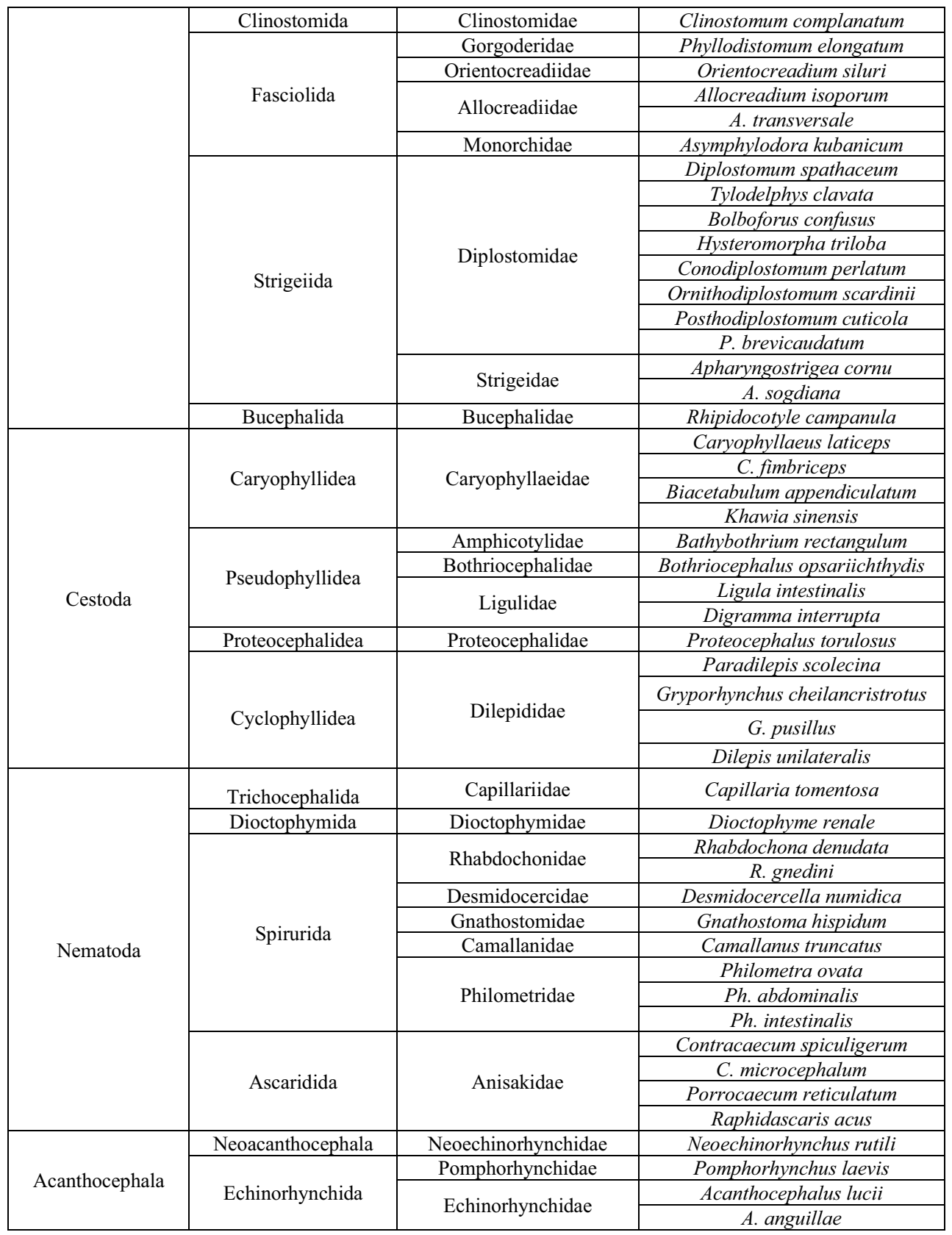

Other orders were represented by one or two species, common helminths of Cypriniformes. Worthy of note are the findings of Dioctophyme renale Goeze, 1782 larvae III in Cypriniformes in the studied region. Previously this species was reported in many fish inhabiting water bodies along the Amydarya River and in the lower reaches of the Syrdarya River [4-7].

The greatest diversity of species of worms turned Syrdarya River waters (39), followed by the Chirchik River (15). The lowest number of helminths was recorded in the AidarArnasay lake system (AALS), where only eight helminth species were found: 3 trematodes, 2 cestodes and 3 nematodes. In our opinion, this is connected with peculiar ecological 
conditions in different water bodies (Table 2). Ten helminth species were found in the fish farms. At the same time prevalence and intensity of infestation varies widely.

Among of the registered helmiths often frequently recorded pathogen species were Sanguinicola inermis Plehn, 1905, Diplostomum spathaceum (Rudolphi, 1819), Tylodelphys clavata (Nordmann, 1832), Khawia sinensis (Hsü, 1935), Bothriocephalus opsariichthydis (Yamaguti, 1934), Ligula intestinalis (Linnaeus, 1758) larvae, Digramma interrupta (Rudolphi, 1810) larvae and Raphidascaris acus (Bloch, 1779) larvae.

The poor diversity of the parasite fauna in Cypriniformes in landlocked water bodies and an almost complete absence of a number of specific parasites are undoubtedly associated with the process of acclimatization, where natural processes of the formation of respective groups of considered helminth hosts were disturbed.

Table 2. Distribution of helminths of Cypriniformes in water bodies of different types of the northeast of Uzbekistan (2009-2020).

\begin{tabular}{|c|c|c|c|c|c|c|c|c|c|c|c|c|c|c|c|c|}
\hline \multirow[b]{2}{*}{$\begin{array}{l}\stackrel{\mathscr{U}}{\varpi} \\
\text { के }\end{array}$} & \multicolumn{4}{|c|}{$\begin{array}{l}\text { Mid-course of the } \\
\text { Syrdarya River }\end{array}$} & \multicolumn{4}{|c|}{ Chirchik River } & \multicolumn{4}{|c|}{$\begin{array}{l}\text { Aidar-Arnasay lake } \\
\text { system }\end{array}$} & \multicolumn{4}{|c|}{ Fish farms } \\
\hline & 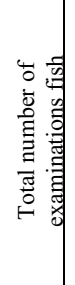 & 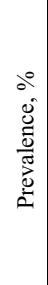 & 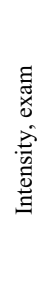 & 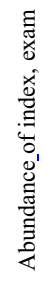 & 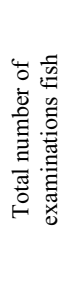 & 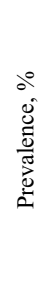 & 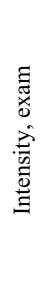 & 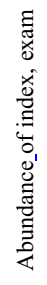 & 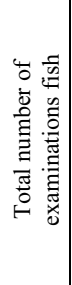 & 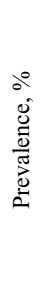 & 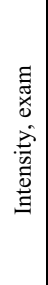 & 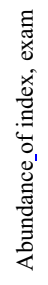 & 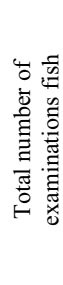 & 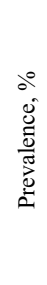 & 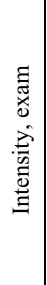 & 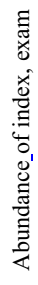 \\
\hline 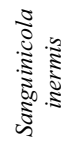 & & $\stackrel{\infty}{-}$ & $\stackrel{\infty}{ \pm}$ & $\widehat{\widehat{c}}$ & & ' & ' & ' & & ' & ' & ' & & ' & ' & ' \\
\hline 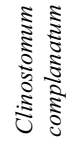 & & & ' & ' & & $\stackrel{\circ}{i}$ & $n$ & $\vec{n}$ & & & ' & ' & & $\vec{i}$ & $\ddot{6}$ & m. \\
\hline 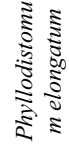 & 8 & & ' & ' & 畣 & ' & ' & ' & $\frac{0}{\infty}$ & ' & ' & ' & 苦 & $\stackrel{\circ}{-}$ & $\stackrel{\infty}{ \pm}$ & $\frac{n}{0}$ \\
\hline 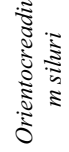 & & & & & & $\stackrel{\circ}{\circ}$ & 㐫 & సे & & & ' & ' & & & ' & ' \\
\hline 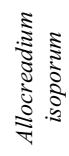 & & $\stackrel{\infty}{\infty}$ & $\stackrel{0}{-1}$ & సి & & $\bar{\sigma}$ & $\stackrel{\infty}{m}$ & లి & & ' & ' & ' & & ' & ' & ' \\
\hline
\end{tabular}




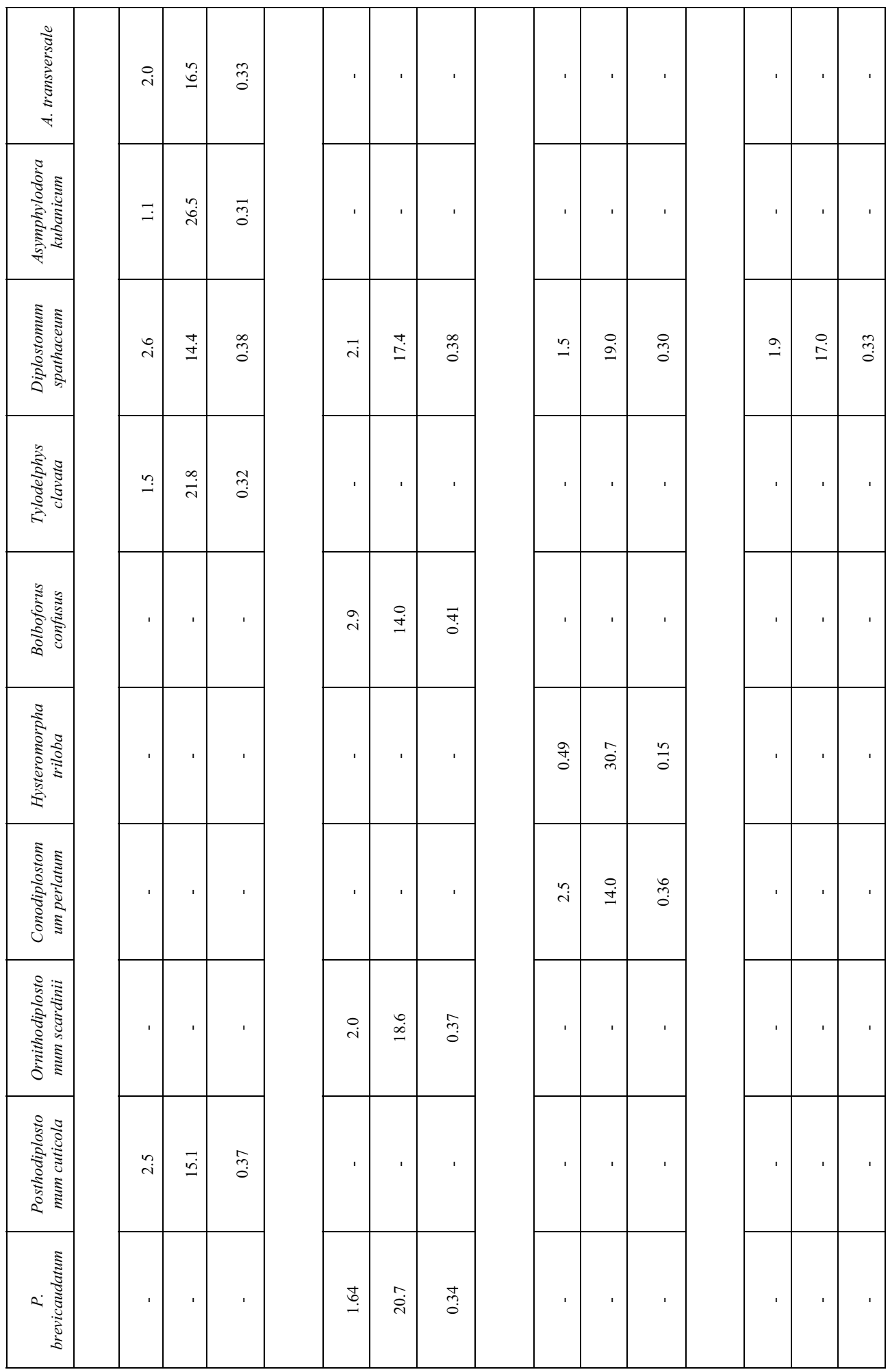




\begin{tabular}{|c|c|c|c|c|c|c|c|c|c|c|c|c|}
\hline 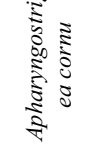 & & ' & ' & $\vec{i}$ & $\stackrel{\circ}{\infty}$ & ले & ' & ' & ' & & ' & ' \\
\hline 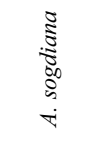 & $\vec{i}$ & $\stackrel{\circ}{\dot{\varphi}}$ & $\stackrel{+}{m}$ & . & ' & ' & ' & ' & ' & ' & ' & ' \\
\hline 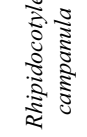 & $\stackrel{n}{n}$ & $\stackrel{\circ}{\stackrel{i}{0}}$ & $\vec{m}$ & ' & ' & , & ' & ' & ' & $\stackrel{\text { Iִ }}{ }$ & $\stackrel{\text { I }}{\mathrm{N}}$ & రి \\
\hline 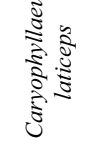 & $\stackrel{\text { Iִ }}{-}$ & $\stackrel{\circ}{=}$ & $\stackrel{m}{0}$ & & & & ' & & ' & & ' & ' \\
\hline 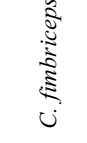 & $\stackrel{m}{\mathrm{I}}$ & : & $\stackrel{t}{0}$ & ' & ' & ' & ' & ' & ' & $\stackrel{+}{\ominus}$ & $n$ & $\stackrel{5}{0}$ \\
\hline 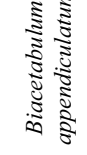 & $\stackrel{0}{\dot{m}}$ & $\stackrel{0}{\circ}$ & $\stackrel{\infty}{0}$ & ' & ' & ' & ' & ' & ' & ' & ' & ' \\
\hline 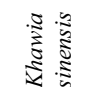 & $\tilde{i}$ & : & $\stackrel{0}{0}$ & $\stackrel{\stackrel{\sim}{i}}{\mathrm{~s}}$ & $\overrightarrow{6}$ & $\stackrel{n}{0}$ & ' & ' & ' & $\stackrel{n}{i}$ & ف & $\stackrel{\circ}{\circ}$ \\
\hline 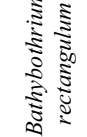 & ' & ' & ' & $\stackrel{\dot{i}}{ }$ & $\stackrel{\sim}{+}$ & $\begin{array}{l}+ \\
\infty \\
0\end{array}$ & ' & ' & ' & $\stackrel{\text { iे }}{0}$ & $\stackrel{\text { I }}{f}$ & $\begin{array}{l} \pm+\infty \\
0 \\
0\end{array}$ \\
\hline 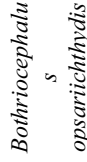 & $\overrightarrow{+}$ & $\stackrel{+}{n}$ & ָี & $\stackrel{+}{+}$ & $\overrightarrow{r i}$ & సี & ' & ' & ' & , & , & ' \\
\hline 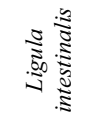 & ${ }^{m}$ & $\vec{\pi}$ & 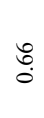 & $\stackrel{n}{=}$ & 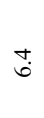 & $\stackrel{m}{0}$ & $\underset{m}{m}$ & $\stackrel{\circ}{\circ}$ & 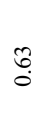 & $\stackrel{n}{=}$ & శై & 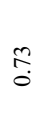 \\
\hline
\end{tabular}




\begin{tabular}{|c|c|c|c|c|c|c|c|c|c|c|c|c|}
\hline 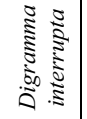 & $\begin{array}{l}\stackrel{\bullet}{\Xi} \\
=\end{array}$ & $\stackrel{\sim}{\underset{f}{*}}$ & $\stackrel{t}{0}$ & $\begin{array}{l}\infty \\
\stackrel{\infty}{\oplus}\end{array}$ & $\stackrel{m}{+}$ & $\stackrel{\substack{\infty \\
0}}{0}$ & & . & ' & $\tilde{a}$ & ț & $\stackrel{\overrightarrow{0}}{\circ}$ \\
\hline 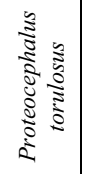 & $\begin{array}{l}\stackrel{\nabla}{ } \\
\dot{\lambda}\end{array}$ & $\overrightarrow{\mathrm{i}}$ & $\stackrel{\infty}{0}$ & . & ' & ' & $\begin{array}{l}\infty \\
\stackrel{\sim}{\sim}\end{array}$ & $\stackrel{\infty}{-}$ & $\vec{n}$ & $\hat{\sigma}$ & $\stackrel{\sim}{f}$ & fี \\
\hline 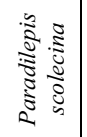 & $\stackrel{\infty}{\stackrel{\infty}{r}}$ & $\begin{array}{l}\bullet \\
+ \\
+\end{array}$ & $\overbrace{0}^{\infty}$ & ' & ' & ' & 1 & ' & ' & ' & ' & ' \\
\hline 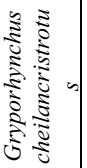 & $\tilde{n}$ & $\begin{array}{l}\dot{n} \\
\dot{n}\end{array}$ & $\stackrel{2}{\stackrel{0}{0}}$ & ' & ' & ' & & . & ' & ' & ' & , \\
\hline 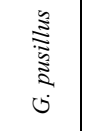 & $\vec{\infty}$ & ชె & $\vec{n}$ & ' & ' & ' & 1 & ' & ' & ' & ' & ' \\
\hline 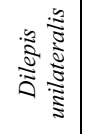 & $\stackrel{m}{+}$ & in & స̃ & ' & ' & ' & 1 & ' & ' & ' & ' & ' \\
\hline 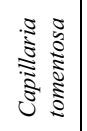 & $\underline{m}$ & in & $\stackrel{r}{\hat{0}}$ & ' & ' & ' & & ' & ' & ' & ' & ' \\
\hline 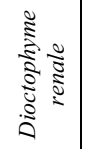 & $\begin{array}{l}\infty \\
\infty\end{array}$ & $\tilde{b}$ & in & ' & ' & ' & $\vec{\infty}$ & గु & \begin{tabular}{l}
\multirow{n}{*}{} \\
0
\end{tabular} & ' & ' & ' \\
\hline 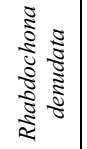 & $\begin{array}{l}\infty \\
\infty \\
\infty\end{array}$ & $\overbrace{b}^{3}$ & in & $\stackrel{?}{\sim}$ & $\hat{\sigma}$ & in & & ' & ' & ' & ' & ' \\
\hline 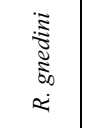 & $\begin{array}{l}\infty \\
\dot{n}\end{array}$ & $\dot{m}$ & $\stackrel{\infty}{+\infty}$ & ' & ' & ' & ' & ' & ' & ' & ' & ' \\
\hline
\end{tabular}




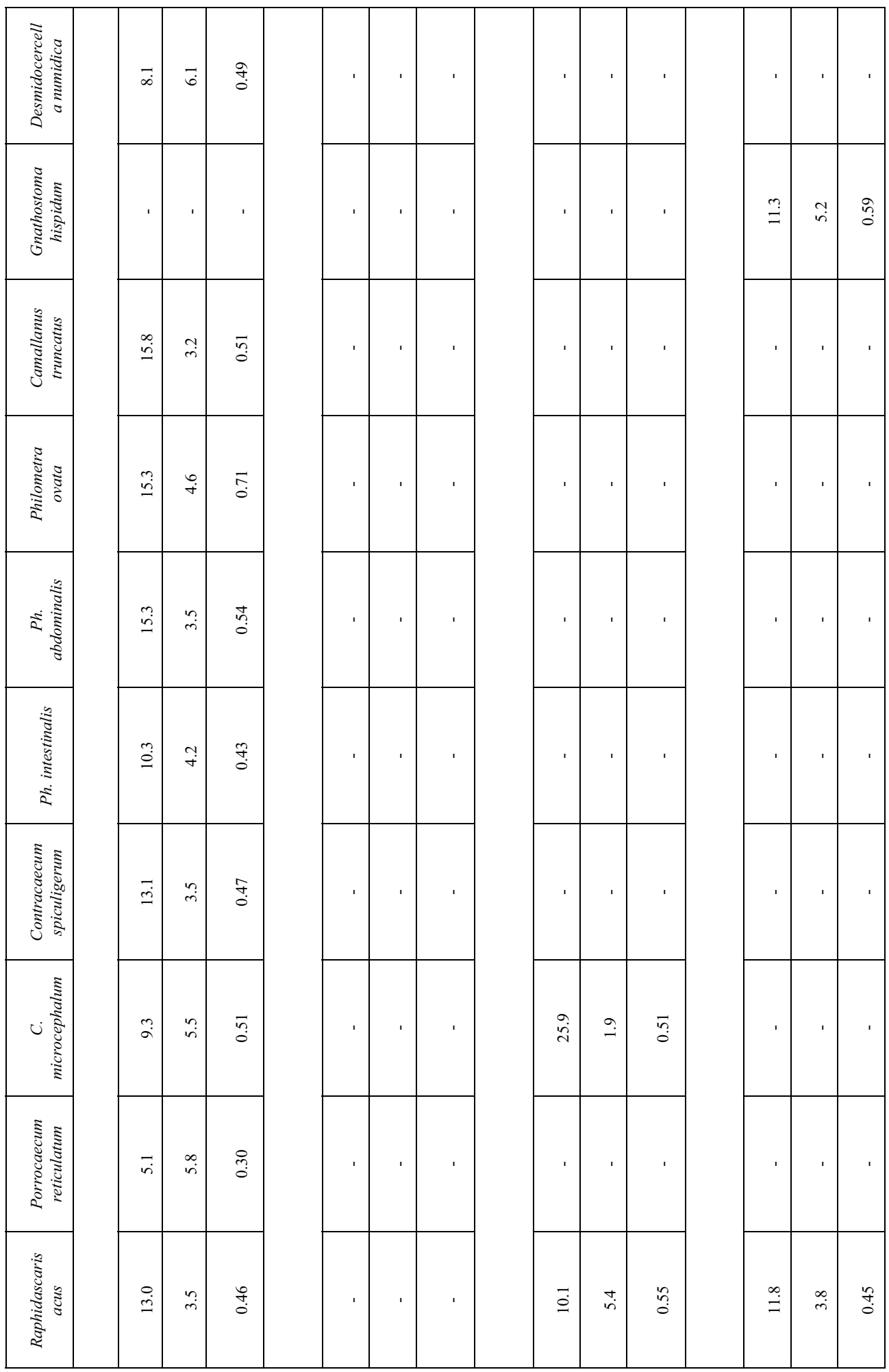




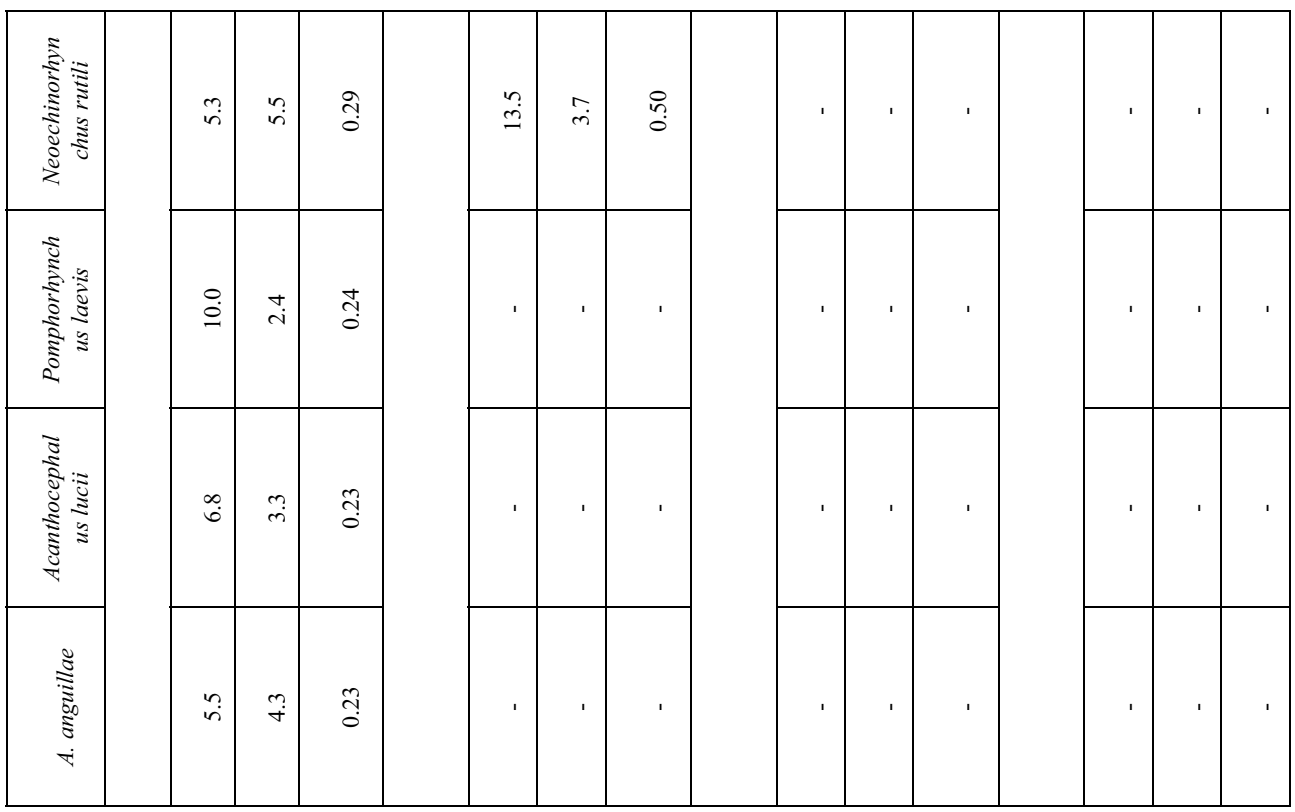

Data we obtained on the quantitative composition of helminth fauna of the Cypriniformes and their biological characteristics make it possible to allocate three types of communities:helminths parasitizing Cypriniformes as definitive hosts; helminths parasitizing Cypriniformes as intermediate hosts; helminths parasitizing Cypriniformes as a reservoir (=paratenetic) hosts;

The distribution of indicated helminth communities in the region depends on a number of well-known biotic and abiotic factors. The first type includes 25 helminth species: 7 trematodes, 7 cestodes, 11 nematodes, and 4 acanthocephalans (Table 3). Fish are infected here mainly through the digestive canals of hosts, as well as directly by a free-swimming nematode larvae (Capillaria tomentosa Dujardin, 1843) and penetration of trematode cercariae (Sanguinicola inermis Plehn, 1905) through the cover of cypriniform fish. There is no uniform opinion (Ginetsinskaya, 1958; Mozgovoy, Kosinova, 1963; Engashev, 1965; Moravec, 1970; Smith, 1984, cited by Pugachev, 2004) regarding the participation of different categories of hosts (both intermediate and paratenic) in the recorded nematodes of the family Anisakidae Skrjabin et Karokhin, 1945: Raphidascaris acus (Bloch, 1779), Porrocaecum reticulatum (Linstow, 1890), Contracaecum spiculigerum (Rudolphi, 1809) and C. microcephalum (Rudolphi, 1819). Views of the authors are contradictory, sometimes excluding one another.

Nevertheless, we find acceptable the viewpoints of Ginetsinskaya (1958) and Engashev (1965) [4-10], who consider Cypriniformes as second intermediate hosts of Raphidascaris acus. We also find this view reasonable for the species of Porrocaecum and Contracaecum.

The second type is characterized by the fact that some species of Cypriniformes are the second intermediate hosts for nineteen helminth species: 12 trematodes, 2 cestodes and 5 nematodes. Definitive hosts (predatory fish, fish-eating birds and mammalians) are infected consuming Cypriniformes fish infected by helminth larvae. 
Table 3. Biological characteristics of helminths of Cypriniformes in the studied area.

\begin{tabular}{|c|c|c|c|c|c|}
\hline \multirow{3}{*}{ Helminths } & \multirow{3}{*}{$\begin{array}{l}\text { No. of } \\
\text { species }\end{array}$} & \multicolumn{4}{|c|}{ Hosts } \\
\hline & & \multicolumn{2}{|c|}{ Intermediate hosts } & \multirow{2}{*}{ Reservoir } & \multirow{2}{*}{ Definitive } \\
\hline & & First & Second & & \\
\hline \multicolumn{6}{|c|}{ Trematoda } \\
\hline Bucephalidae & 1 & Mollusks & Cypriniformes & - & $\begin{array}{l}\text { Cypriniformes } \\
\text { and other fish }\end{array}$ \\
\hline Sanguinicolidae & 1 & Mollusks & - & - & $\begin{array}{l}\text { Cypriniformes } \\
\text { and other fish }\end{array}$ \\
\hline Allocreadiidae & 2 & Mollusks & - & - & $\begin{array}{l}\text { Cypriniformes } \\
\text { and other fish }\end{array}$ \\
\hline Gorgoderidae & 1 & Mollusks & - & - & $\begin{array}{l}\text { Cypriniformes } \\
\text { and other fish }\end{array}$ \\
\hline Monorchidae & 1 & Mollusks & - & - & $\begin{array}{l}\text { Cypriniformes } \\
\text { and other fish }\end{array}$ \\
\hline Orientocreadiidae & 1 & Mollusks & - & - & $\begin{array}{l}\text { Cypriniformes } \\
\text { and other fish }\end{array}$ \\
\hline Clinostomidae & 1 & Mollusks & Fish & - & Birds \\
\hline Diplostomidae & 8 & Mollusks & Fish & - & Birds \\
\hline Strigeidae & 2 & Mollusks & Fish & - & Birds \\
\hline \multicolumn{6}{|c|}{ Cestoda } \\
\hline Caryophyllaeidae & 4 & Oligochaetes & - & - & Cypriniformes \\
\hline Amphicotylidae & 1 & Cyclops & - & - & Cypriniformes \\
\hline $\begin{array}{c}\text { Bothriocephalida } \\
\text { e }\end{array}$ & 1 & Cyclops & - & - & Cypriniformes \\
\hline Ligulidae & 2 & Cyclops & Cypriniformes & - & Birds \\
\hline Proteocephalidae & 1 & Cyclops & - & - & Cypriniformes \\
\hline Dilepididae & 4 & Cyclops & - & - & Birds \\
\hline \multicolumn{6}{|c|}{ Nematoda } \\
\hline Capillariidae & 1 & - & - & - & $\begin{array}{l}\text { Cypriniformes } \\
\text { and other fish }\end{array}$ \\
\hline Dioctophymidae & 1 & Oligochaetes & Cypriniformes & - & Mammalians \\
\hline Rhabdochonidae & 2 & Oligochaetes & - & - & Cypriniformes \\
\hline Desmidocercidae & 1 & - & - & Cypriniformes & Birds \\
\hline Camallanidae & 1 & Cyclops & - & - & Cypriniformes \\
\hline Philometridae & 3 & Cyclops & - & - & Cypriniformes \\
\hline Gnathostomatidae & 1 & Cyclops & - & $\begin{array}{c}\text { Cypriniformes, } \\
\text { amphibians } \\
\end{array}$ & Mammalians \\
\hline Anisakidae & 4 & $\begin{array}{l}\text { Oligochaetes } \\
\text { and Copepoda }\end{array}$ & $\begin{array}{c}\text { Cypriniformes, } \\
\text { dragonflies }\end{array}$ & Cypriniformes & $\begin{array}{c}\text { Birds, predatory } \\
\text { fish } \\
\end{array}$ \\
\hline \multicolumn{6}{|c|}{ Acanthocephala } \\
\hline $\begin{array}{l}\text { Neoechinorhyn- } \\
\text { chidae }\end{array}$ & 1 & Ostracoda & $\begin{array}{c}\text { Megaloptera, } \\
\text { Hirudinea }\end{array}$ & - & Cypriniformes \\
\hline Echinorhynchidae & 2 & Amphipoda & - & - & $\begin{array}{l}\text { Cypriniformes } \\
\text { and other fish }\end{array}$ \\
\hline $\begin{array}{c}\text { Pomphorhynchid } \\
\text { ae }\end{array}$ & 1 & Amphipoda & - & - & $\begin{array}{l}\text { Cypriniformes } \\
\text { and other fish }\end{array}$ \\
\hline
\end{tabular}

Participation of Cypriniformes as paratenic hosts in the transmission of the considered helminths is in many respects questionable. Nevertheless, Cypriniformes, according to literature, were noted as paratenetic hosts [3].

According to our findings, they are noted for two nematode species of the genera Desmidocercella and Gnathostoma, which should be assigned to the third type.

According to our findings, Cypriniformes in the studied region promote the circulation of a number of cestode, trematode, nematode and acanthocephalan species in predatory fishes, birds and mammalians (Table 3). 
Distribution of helminths of Cypriniformes in some parts of the Syrdarya River basin is not equivalent. The optimal conditions for the functioning of the communities of helminths obviously represented in the reservoirs of the middle reaches, where we recorded 49 species of parasites. In the 60 years of the last century, in this region have been reported 20 species of helminths $[1,3]$. In other parts of the Syrdarya noted significantly fewer species, 25 - in the lower reaches $[4,9]$ and 10 - in the headwaters of $[2,5]$.

\section{Conclusions}

Carried research has shown that qualitative and quantitative distribution of helminths Cypriniformes in water bodies of the basin of the Syrdarya River is very uneven. The greatest diversity of species is characterized by well warmed water bodies in coastal parts of the river. Here are optimal conditions for the development of parasites Cypriniformes and attract birds and mammals involved in the circulation of helminths.

Species diversity of helminths Cypriniformes, in the investigated region is rich enough and stable functions in water biocenosis. The parasitic worms include 49 species of Cypriniformes: 18 species of Trematoda, 13 of Cestoda, 14 of Nematoda and 4 of Acanthocephala. Among the species mentioned most common pathogenic representatives of the family Sanguinicolidae, Diplostomidae, Bothriocephalidae, Ligulidae and Anisakidae, which a negative affect the development of hamper the fishing industry of the country. This fact dictates conduct of involves constant monitoring of ichthyoparasitological situation in specific natural and artificial water bodies of Uzbekistan.

We are very grateful to Professor, Department of bio-ecology of the Far Eastern State Technical University, Ph.D. T.E. Butorina for advice in determining helminth Cypriniformes fishes of Uzbekistan.

\section{References}

1. O. Bauer, E. Skrjabina, Parasitic multicellular, 3, 311-339 (1987)

2. I. Byhovskaya-Pavlovskaya, Parasites of fishes, 121 (1985) (in Russian)

3. O. Pugachev, Parasitology, 36(1), 3-10 (2002)

4. O. Pugachev, Proceedings of the Zoological Institute of the Russian Academy of Sciences, 298, 218-225 (2003)

5. O. Pugachev, Proceedings of the Zoological Institute of the Russian Academy of Sciences, 304, 244-250 (2004)

6. F. Safarova, U. Shakarbaev, F. Akramova, V. Golovanov, Journal of Russian parasitology, 1, 44-48 (2014)

7. A. Shigin, Nauka, 253 (1986) (in Russian)

8. I. Khokhlova, Nauka, 276 (1986) (in Russian)

9. F. Moravec, Folia parasitol, 27(4), 347-348 (1980)

10. F. Moravec, Parasitic nematodes of freshwater fishes of Europe, 473 (1994)

11. J. Smith, Can. J. Zool., 62, 1378-1386 (1984)

12. Y. Li, K. Cao, C. Fu, Mitochondrial DNA Part B, 3(2), 803-804 (2018)

13. P. Mathews, Journal of Experimental Zoology, India, 22(2), 713-718 (2019)

14. W. Chen, S. Zhu, X. Li, Y. li, Conservation Genetics Resources, 1-7 (2021) 